\title{
Experimental Investigation of the Diagnosis of Angular Contact Ball Bearings Using Acoustic Emission Method and Empirical Mode Decomposition
}

\author{
Ramtin Tabatabaei, ${ }^{1}$ Aref Aasi $\mathbb{D}^{1,2}$ and Seyed Mohammad Jafari ${ }^{1}$ \\ ${ }^{1}$ Faculty of Mechanical and Energy Engineering, Shahid Beheshti University, Tehran, Iran \\ ${ }^{2}$ Department of Mechanical Engineering, Worcester Polytechnic Institute, Worcester, MA 01609, USA \\ Correspondence should be addressed to Aref Aasi; aaasi@wpi.edu
}

Received 10 January 2020; Revised 30 July 2020; Accepted 15 September 2020; Published 30 September 2020

Academic Editor: Enrico Ciulli

Copyright (c) 2020 Ramtin Tabatabaei et al. This is an open access article distributed under the Creative Commons Attribution License, which permits unrestricted use, distribution, and reproduction in any medium, provided the original work is properly cited.

Early detection of angular contact bearings, one of the important subsets of rolling element bearings (REBs), is critical for applications of high accuracy and high speed performance. In this study, acoustic emission (AE) method was applied to an experimental case with defects on angular contact bearing. AE signals were collected by AE sensors in different operating conditions. Signal to noise ratio (SNR) was calculated by kurtosis to entropy ratio (KER), then acquired signals were denoised by empirical mode decomposition (EMD) method, and optimal intrinsic mode function (IMF) was selected by the proposed method. Finally, envelope spectrum was applied to the denoised signals, and frequencies of defects were obtained in different rotating speeds, loadings, and defect sizes. For the first time, a small defect with width of $0.3 \mathrm{~mm}$ and loading of $475 \mathrm{~N}$ was detected in early stage of $0.04 \mathrm{KHz}$. Moreover, a comparison between theoretical and extracted defect frequencies suggested that our method successfully detected localized defects in both inner and outer race. Our results show promise in detecting small size defects in REBs.

\section{Introduction}

Rolling element bearings (REBs) are widely used in rotating machines and in various industries such as steel, mining, paper, and railways [1]. Specifically, angular contact bearings (in this paper) have many applications such as vacuum pumps, machine components for semiconductor industry, high-speed rolling mills, high-precision machine tools, printing machineries [2]. Since it can be used in very accurate applications at high speeds (such as the shafts in process pumps), it is critical case and necessary for early detection of failure in the early stages. Failure of bearings is a major cause of machinery breakdown, economic losses, and even loss of human lives. Undesirable vibrations can be caused by faulty installation, poor maintenance, or surface spall that finally leads to development of REB failure [3].

There is enormous amount of research in the field of diagnosis of REBs [4]. One of the important issues is how to identify bearing fault before it reaches the final failure state. Bearing failure is reported to account for almost $40 \%-50 \%$ of motor failure cases in industries [5]. Several methods have been applied in diagnosis of REBs such as vibration analysis, thermographic inspection, and nondestructive testing (NDT) techniques like acoustic emission (AE). AE technology is superior to other methods in detecting defects in early stages. Another advantage of using of AE is the discovery of defects in slow-speed and extremely slow-speed bearings that cannot be detected through other methods $[6,7]$.

Balderston used the AE method in the diagnosis of bearings for the first time [8]. One of the methods for analyzing the signal in diagnosis of REBs is the use of $\mathrm{AE}$ parameters and indicators in discovering such cases as location of defects, size, and defect growth, taking into account the sensitivity of AE parameters to operating speeds and loading. Examples of these parameters and indicators 
include counting parameter, peak amplitude, energy, rise time, time, RMS, skewness, kurtosis, crest factor (CF).

Yoshioka and Fujiwara [9] did a research on fatigue of rolling contact bearings and claimed that AE measurements can make it possible to understand the entire failure process. Rogers [10] inspected slowly rotating bearings for gas extraction cranes using $\mathrm{AE}$ method and kurtosis indicator. $\mathrm{He}$ used this method to detect defects in the early stages and locate the fatigue cracks. By comparing the results of $\mathrm{AE}$ test with vibration analysis, he showed that the vibration analysis was difficult due to the low speed of the crane bearings and it was not possible to detect defects in the early stages.

Tandon and Nakra [11] studied AE method in REBs by making artificial defects on inner, outer, and rolling elements and concluded that the peak amplitude of frequency of signals is higher than healthy bearing in various operating conditions, but they could not detect faults in slow-speed bearings. Tandon and Choudhury [12] reviewed the methods of vibration and AE in diagnosis of REBs and stated that the $\mathrm{AE}$ has typical frequency content in the range of $100 \mathrm{kHz}$ to $1 \mathrm{MHz}$, so $\mathrm{AE}$ is not influenced or distorted by unbalancing or misalignment which are at low-frequency ranges. The $\mathrm{AE}$ technique and $\mathrm{AE}$ parameters have a significant advantage over vibration measurement methods due to their high sensitivity to the detection of the incipient bearing faults. Choudhury and Tandon [13] investigated defects on inner race and rolling elements by AE method, and they chose counting and peak amplitude parameters of AE for detection of defects. They argued that counting parameters can detect and peak amplitude can show the size of defects, but they did not investigate defects on outer race of REBs.

Mba [14] studied the defects in the inner and outer race of a radially roller bearing, considering two RMS and counting parameters in different operating conditions by changing rotational speed and load and considering two types of defects, small and large. He chose an appropriate threshold level for AE count and emphasized that selection of an appropriate threshold depends on the experience of investigator and type of system. His experiments showed that upon increasing the speed, the load and also the small and large defects of the outer race can cause an increase in the RMS value. However, in the case of defects in the inner race, the same trend was not observed. Mba and Rao [15] reviewed $\mathrm{AE}$ applications for condition monitoring and diagnosis of different rotating machines including bearings.

Rahman et al. [16] investigated the application of AE to monitor rolling contact fatigue on a bearing. They did tests using a test rig consisting of just a ball instead of an entire rolling bearing running; the tests were carried out under constant load and speed for detecting the incipient damage and damage location. They concluded that AE counting parameter is an important parameter for the detection of incipient damage, but their system was simpler and the fault detection complexity was reduced.

In real industrial environment due to high temperatures, rotating speeds, and pressures, desired signals for diagnosis by AE method are always masked by high levels of noise [17]. Hence, to enhance signal to noise ratio (SNR) of AE signals, utilization of adaptive signal processing techniques is important. Therefore, there are numerous denoising techniques for noise reduction in the $\mathrm{AE}$ signal including Hilbert-Huang transform, spectral kurtosis, morphological filters, and wavelet transform (WT) [18-21]. One of the most important and widely used tools for denoising and processing of signals in the time-frequency domain is the wavelet transform, which has been used successfully in many studies, alone or in combination with other signal processing techniques.

Antoni and Randall [22] suggested the use of spectral kurtosis (SK) for detecting and characterizing transient signals buried in additive noise. Discrete wavelet transform (DWT) is widely used in signal denoising of REBs due to its high resolution in time and frequency domains [23]. However, Amiri and Asadi [24] argued that, due to the fact that only the approximated component at each level is decomposed by using the dyadic filter bank, the results of frequency resolution in higher-level DWT decomposition are less accurate. Therefore, the wavelet packet transform (WPT), which is a generalization of wavelet transform (WT) and DWT, offers better denoising ability in nonstationary signals such as defected bearings.

Hao et al. [25] did reduce noises and process the AE signals obtained from a roller bearing with various types of defects, by means of continuous wavelet transform (CWT). They applied this CWT to denoised signals and obtaining the time-frequency spectrum (scalogram) and obtained dominant frequencies that indicate the fault in the bearing.

Parizi et al. [26] studied the statistical parameters such as kurtosis, crest factor (CF), energy, and counting, before and after denoising of the signals with the help of WPT to detect defects of bearings. They reported that the calculation of the statistical parameters after the removal of low frequency noise by means of a WPT showed that the values of the parameters for the defective bearing are more than normal, and the kurtosis is the most appropriate parameter compared to others for detection of defects in REBs. Selection of the mother wavelet plays an important role in reducing the noise of AE signals by means of WT; there are variety of methods for this purpose such as correlation coefficient, variance, and energy to entropy ratio. Rodrigues and DaAZMello [27] used these methods to select optimal mother wavelet for nonstationary signals like bearings.

Recently, there are new methods based on optimization of denoising tool such as combination of WPT and kurtosis to entropy ratio (KER) by Hemati et al. [28], WPT and SNR of the output spectrum by Chacon et al. [29], empirical mode decomposition (EMD) and fast kurtogram by Fu et al. [30], and optimized kurtogram method by Lio et al. [31] which combines kurtogram, Shannon entropy, and autocorrelation function to identify defects in AE signals.

Besides the application of EMD in rotating machines, many investigations emphasized the effectiveness of the EMD method for denoising and obtaining desired features from other signals. For example, EMD has an application in biomedical signal processing. Bajaj and Pachori [32] used intrinsic mode function (IMF) for extracting electroencephalography (EEG) features and then classified seizure 
and nonseizure EEG signals by applying least squares support vector machine (LS-SVM). Pryia et al. [33] used EMD for the classification of alcoholic and normal EEG signals. Jain et al. [34] used Riemann-Liouville fractional integral and EMD for electrocardiograph (ECG) denoising.

In this paper, an experiment is conducted to fault diagnosis on angular contact bearings using acoustic emission technology. An artificial line defect is considered in two different sizes (small and large). At first, signals of the bearing are extracted by $\mathrm{AE}$ sensors at different conditions, and then denoising of the AE signals is investigated. In the proposed method, after preprocessing, $\mathrm{AE}$ signal is decomposed by empirical mode decomposition (EMD), and to obtain better results, the best intrinsic mode function (IMF) based on kurtosis to entropy ratio is selected. After obtaining the desired signal, by using envelope spectrum, frequency of faults is detected. The proposed method shows good accuracy in the fault detection, and the results prove its capability for practical applications.

\section{Method}

In defective rolling element bearings that are in operation, contact of rolling element with defect in inner or outer race produces AE signals (AE hits) [35]. Other reasons which lead to producing AE signals in REBs are friction and wear between components, shortage or lack of lubricant in the bearings, and also contamination of lubricant [36-38]. In this research, artificial faults caused on inner and outer race of the bearing produce AE signals. The schematic of the intended method in this paper is depicted in Figure 1.

\section{Experimental Setup}

A test rig for diagnosis of angular contact bearings is used as shown in Figure 2. The schematic of test rig is also shown in Figure 3. In this experiment, seven bearings have been tested, and experiments on intact and faulty bearings have been done. The conditions depend on speed, load, defect size, and type of sensor. Rotating speed is $600,900,1200$, or $1500 \mathrm{rpm}$.

The type of bearing is $7202 \mathrm{BEP}$ (SKF), which is angular contact with single row, and it can carry combined loads (radial and axial loads).

Artificial defects caused in two different sizes on inner and outer race of bearings are shown in Figure 4; all defects were caused by electrical discharge machining (EDM) method. The specifications of defects are shown in Table 1; four different load sizes were imposed on REBs, with load types including constant axial and radial (Table 2). At each load, four different rotating speeds were surveyed and data collected. The bearings are mounted inside rigid housings that can be preloaded using screws embedded in outer surface of housing.

Data were acquired with two dual-channel cards (4 channels in total), and data rate of each channel is 2 million dots per second. The card type is PAC: PCI-2 with 18 bit A/ $D$ resolution, made by PAC company. Preamplifiers type is PAC: $2 / 4 / 6 \mathrm{~dB}$, and the data acquisition software, made by AEwin company, has a complete and reliable connection to

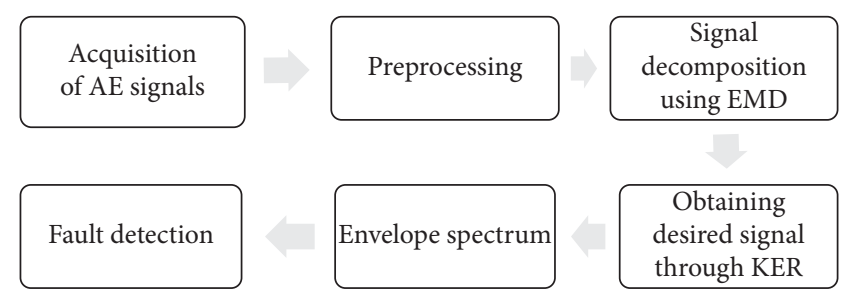

Figure 1: Process of the method.

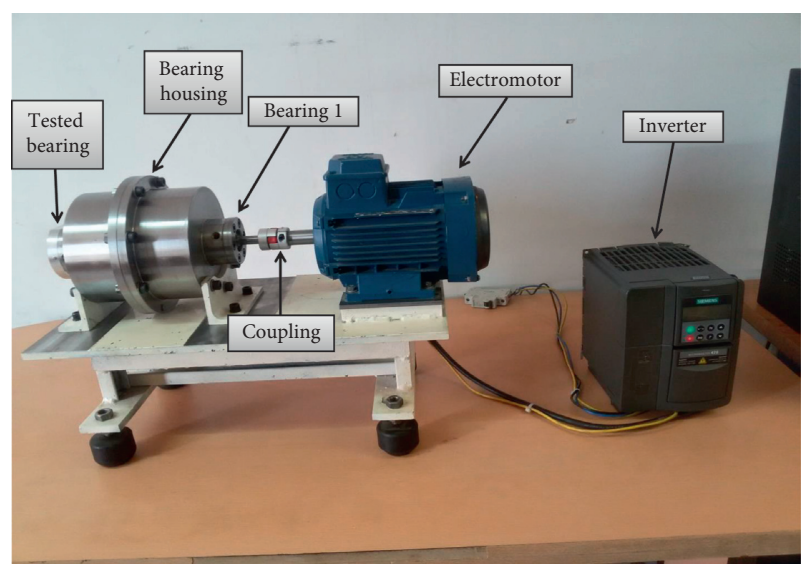

FIGURE 2: Experimental setup.

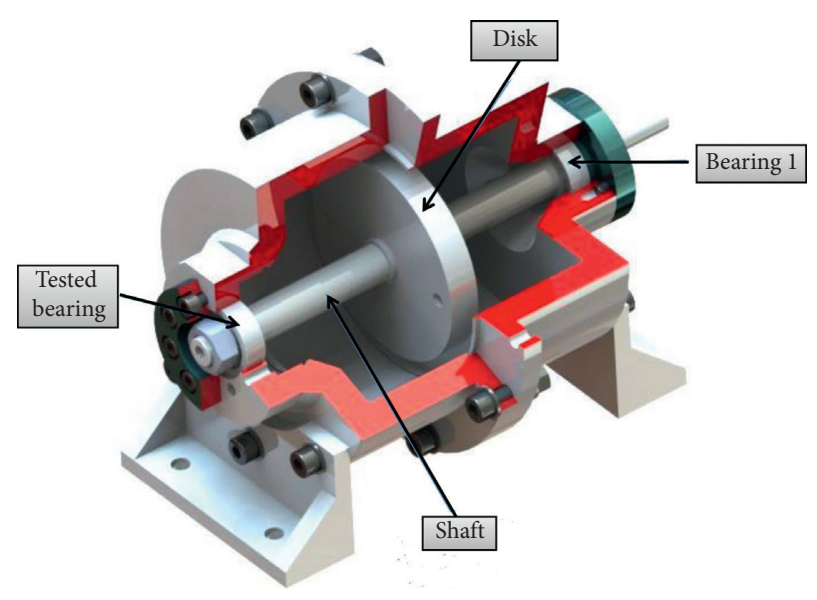

FIgURE 3: Schematic of test rig.

the equipment. The setup with sensors and preamplifiers is shown in Figure 5. The sensor, which is used for signal acquisition, is wideband sensor, and it is shown in Figure 6. Furthermore, preloading is done by tightening the left side locking plate screws and torque wrench applying desirable loads to the locking plate screws as shown in Figure 6.

\section{SNR of Raw AE Signals}

The raw AE signals for different sizes of defects on outer race of the bearing in different conditions have been extracted, and some examples of them are plotted in Figure 7. 


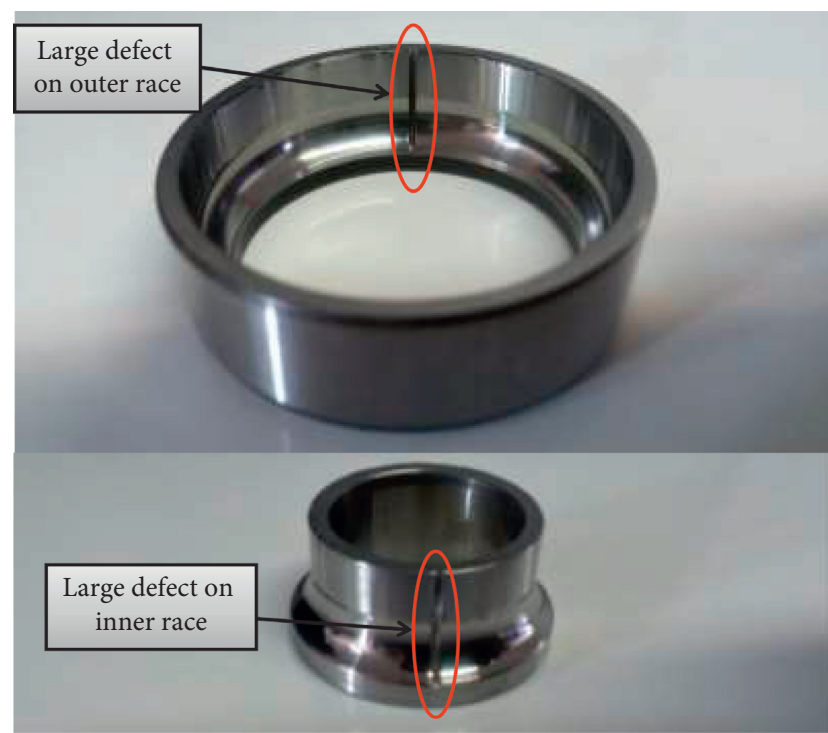

FIGURE 4: Defects caused in outer race of the bearing.

TABLE 1: Specifications of defects.

\begin{tabular}{lcc}
\hline Type of defects & Line defect & Line defect \\
\hline Size of defect & Small defect (SD) & Large defect (LD) \\
Width of defect $(\mathrm{mm})$ & 0.3 & 0.6 \\
\hline
\end{tabular}

Table 2: Different loads imposed on the bearing.

\begin{tabular}{lcccc}
\hline Type of loads & $\begin{array}{c}\text { Small } \\
(\mathrm{SL})\end{array}$ & $\begin{array}{c}\text { Medium- } \\
\text { low (MLL) }\end{array}$ & $\begin{array}{c}\text { Medium- } \\
\text { high (MHL) }\end{array}$ & $\begin{array}{c}\text { High } \\
(\mathrm{HL})\end{array}$ \\
\hline $\begin{array}{l}\text { Imposed } \\
\text { momentum (N.M) }\end{array}$ & 0.7 & 1.7 & 2.7 & 3.7 \\
$\begin{array}{l}\text { Axial load imposed } \\
\text { on bearing (N) }\end{array}$ & 400 & 971 & 1543 & 2114 \\
$\begin{array}{l}\text { Radial load } \\
\text { imposed on } \\
\text { bearing (N) }\end{array}$ & 475 & 1153 & 1832 & 2510 \\
\hline
\end{tabular}

As you can see, by increasing the load and speed, the AE signal created by the defect is not visually detectable as the signal to noise ratio (SNR) increased. To investigate the SNR, a proper indicator called kurtosis to entropy ratio (KER) is proposed. In the signals obtained from the defective bearing, the lower the entropy of a signal, the higher and more periodical the concentration of energy, and the higher the SNR. The kurtosis also examines peaks of signal. It gives a measurement of the degree of impulsiveness and peakiness of the signal $[10,28]$. Therefore, the use of these two indicators can be a good measure to examine the SNR in received signals as follows:

$$
\mathrm{KER}=\frac{\text { Kurtosis }}{\text { Entropy }}
$$

Kurtosis is described as follows [39]:

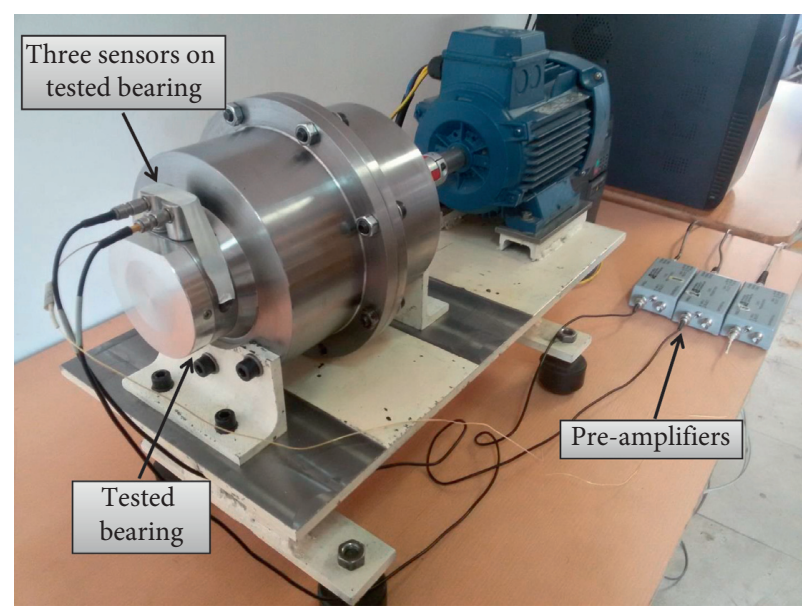

FIgURE 5: The setup with sensors and preamplifiers.

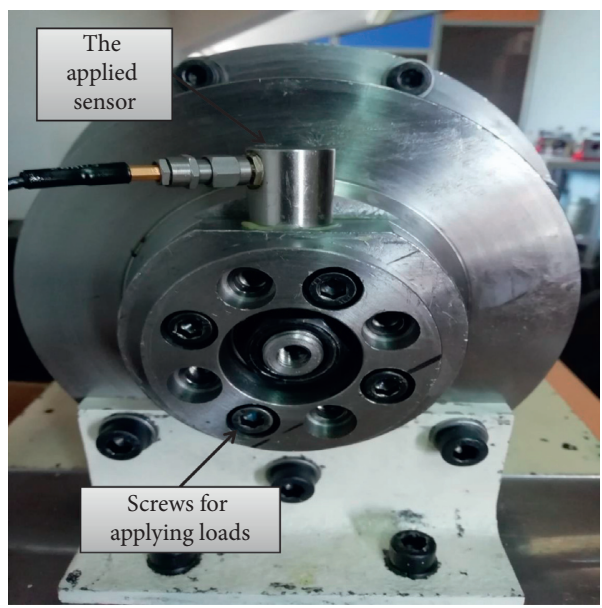

FIGURE 6: The applied sensor and loading system and screws.

$$
K=\frac{N \sum_{i=1}^{N}\left(x_{i}-\bar{x}\right)^{4}}{\left[\sum_{i=1}^{N}\left(x_{i}-\bar{x}\right)^{2}\right]^{2}}
$$

where $x_{i}$ is $i$ th sample of signal, $\bar{x}$ is the mean of samples, and $N$ represents the number of samples.

In addition, entropy is Shannon entropy [40]:

$$
\begin{aligned}
\text { entropy } & =-\sum_{i=1}^{N} P_{i} \times \log P_{i}, \\
\sum_{i=1}^{N} P_{i} & =1,
\end{aligned}
$$

where $P_{i}$ is the distribution of the energy probability:

$$
P_{i}=\frac{\left(x_{i}\right)^{2}}{\sum_{i=1}^{N}\left(x_{i}\right)^{2}}
$$

The results of KER for outer race defect at different conditions are shown in Table 3. 


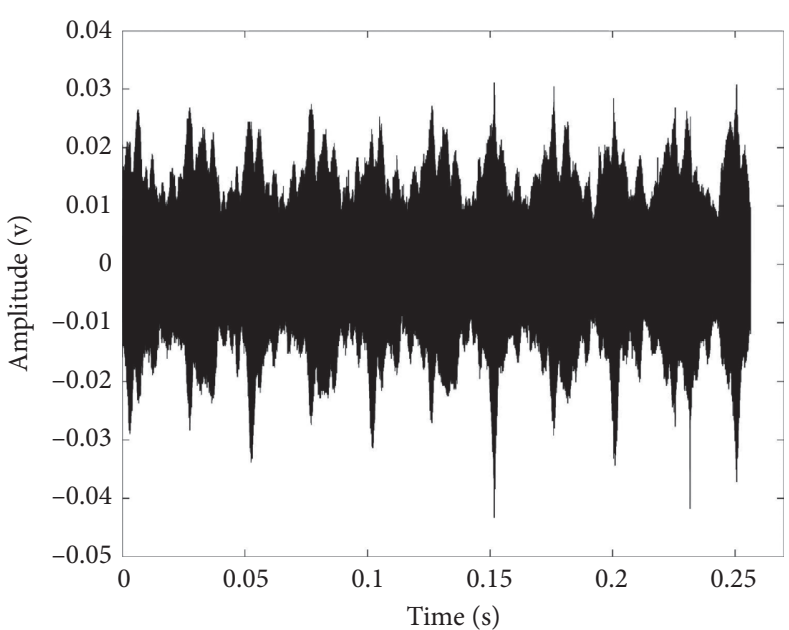

(a)

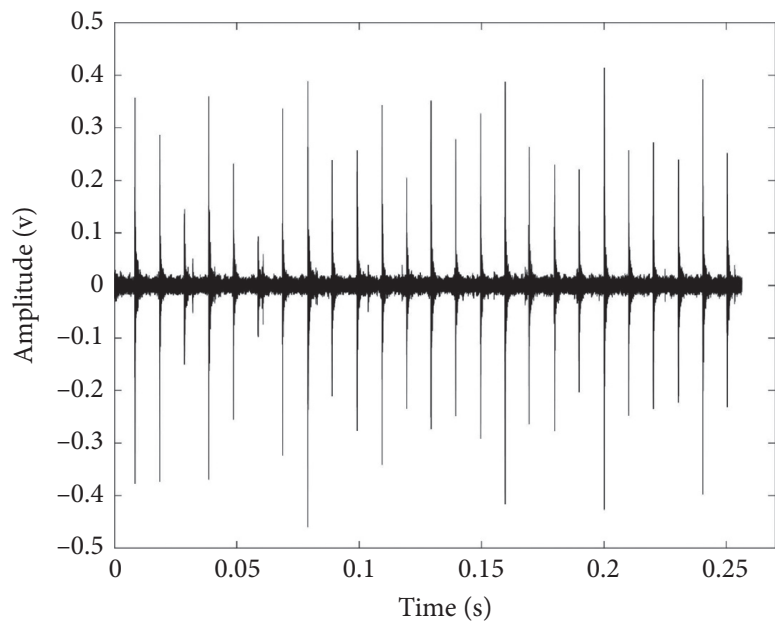

(c)

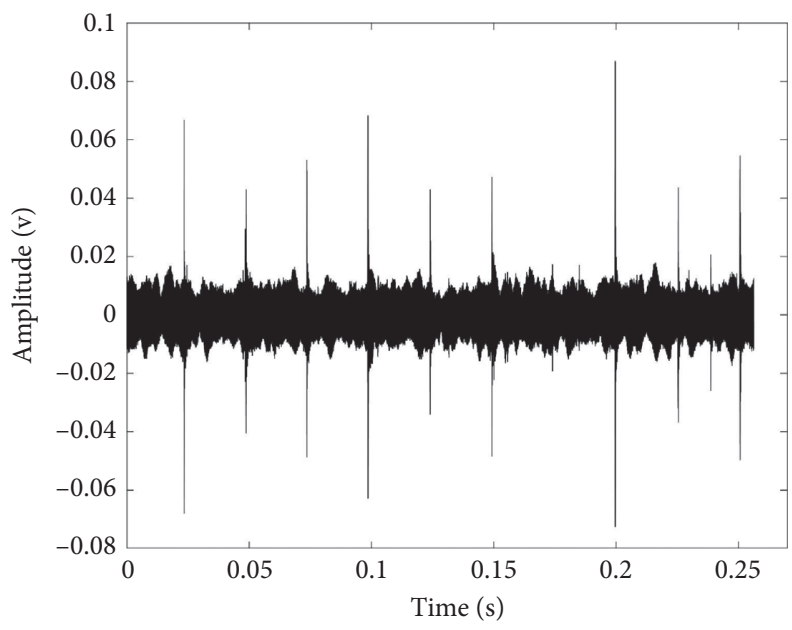

(e)

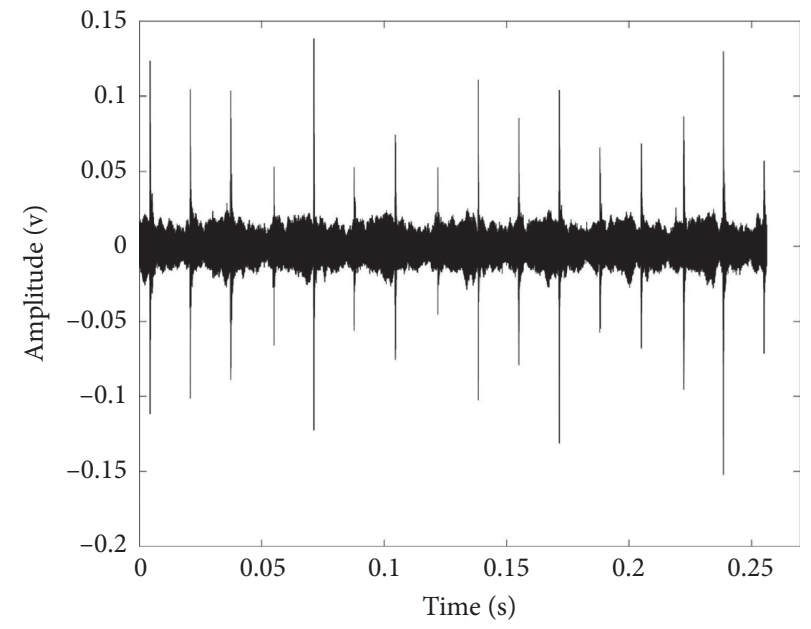

(b)

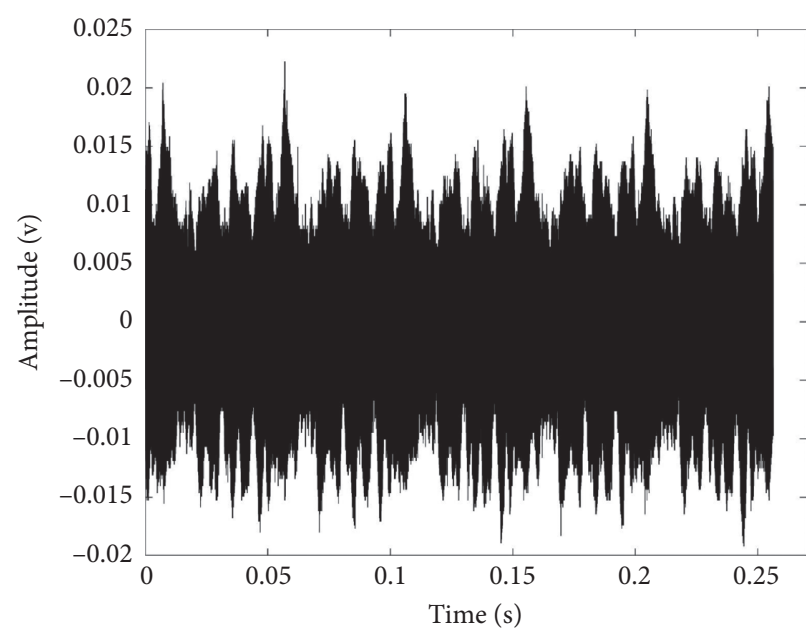

(d)

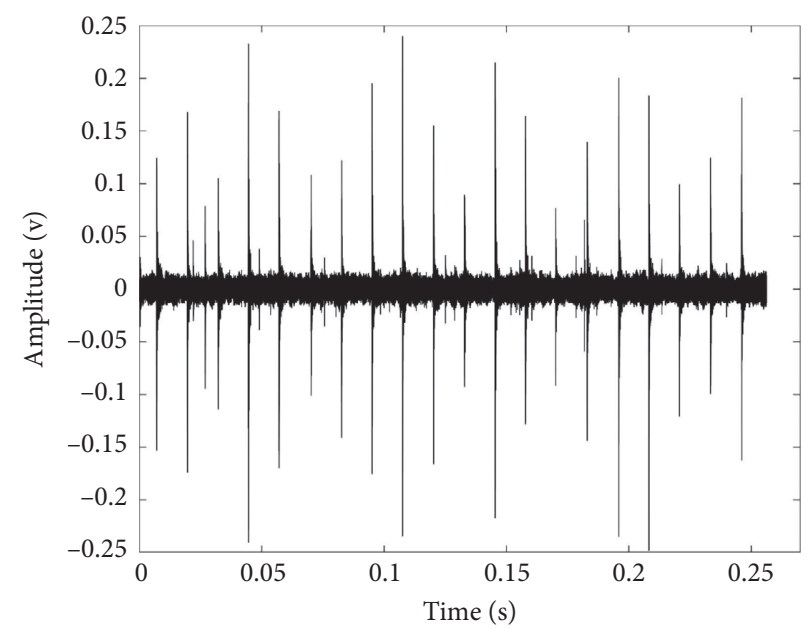

(f)

Figure 7: (a) Small defect, small load, and $600 \mathrm{rpm}$. (b) Small defect, medium-low load, and $900 \mathrm{rpm}$. (c) Small defect, medium-high load, and $1500 \mathrm{rpm}$. (d) Large defect, small load, and $600 \mathrm{rpm}$. (e) Large defect, medium-high load, and $600 \mathrm{rpm}$. (f) Large defect, high load, and $1200 \mathrm{rpm}$. 
TABLE 3: Value of KER for outer race defect at different conditions.

\begin{tabular}{lccc}
\hline KER & Speed $(\mathrm{rpm})$ & Small defect & Large defect \\
\hline & 600 & 0.0126 & 0.0217 \\
Small load & 900 & 0.0114 & 0.0219 \\
& 1200 & 0.0247 & 0.0661 \\
& 1500 & 0.0825 & 0.2507 \\
Medium-low load & 600 & 0.0203 & 0.0273 \\
& 900 & 0.0457 & 0.0636 \\
& 1200 & 0.0947 & 0.2398 \\
Medium-high load & 1500 & 0.1412 & 0.2474 \\
& 600 & 0.0506 & 0.0499 \\
& 900 & 0.0441 & 0.2244 \\
High load & 1200 & 0.0926 & 0.2426 \\
& 1500 & 0.1190 & 0.2204 \\
\hline
\end{tabular}
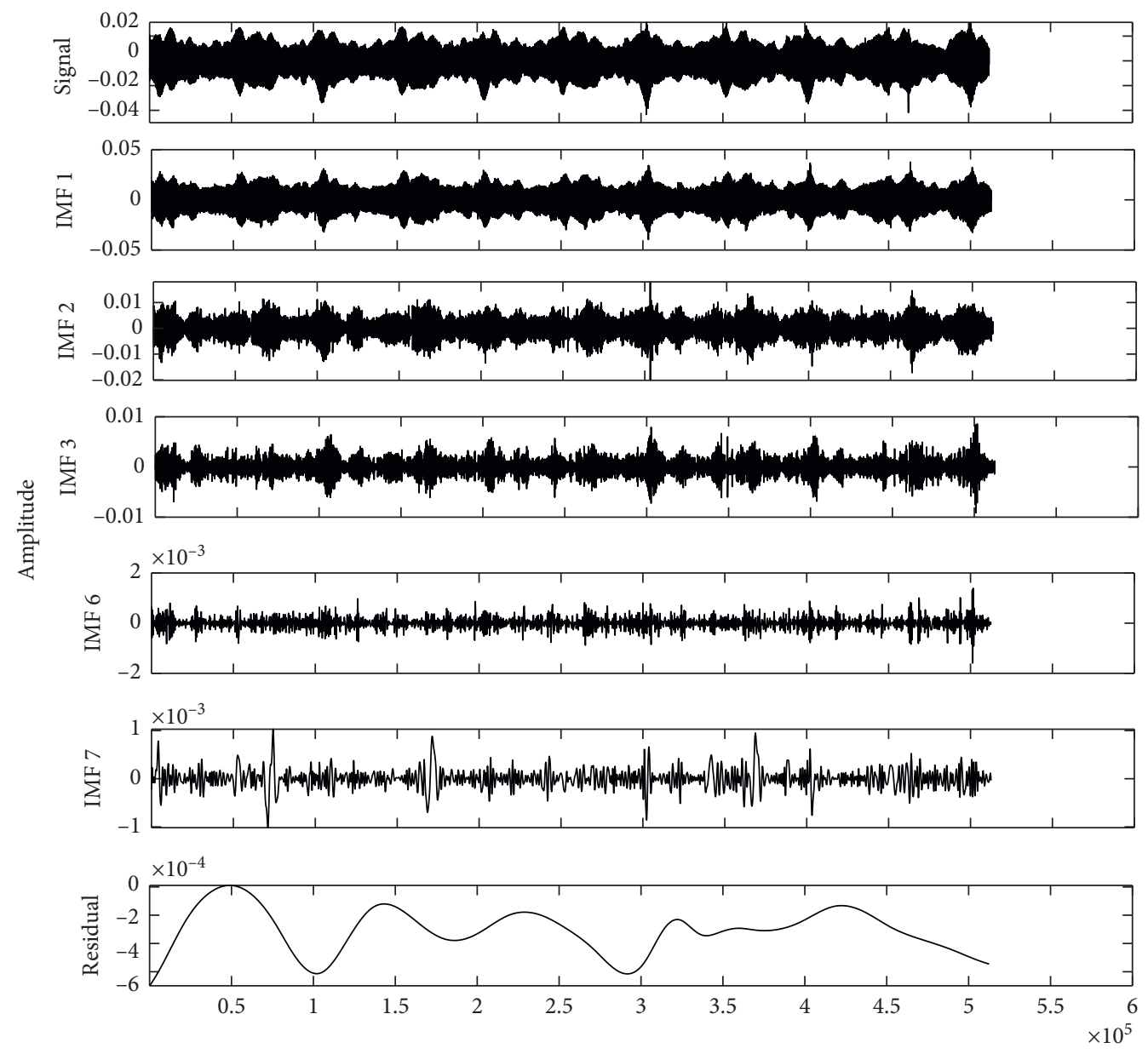

Figure 8: Decomposition of the signal into different IMFs.

From both Figure 7 and Table 3, we can conclude that, by increasing the load and speed, the KER as a measuring tool of SNR is increased and defects can be detected easily, but in low SNR signals it is difficult to detect defects in both the time signal and the frequency spectrum. Hence, the denoising of AE signals is necessary.

\section{Signal Denoising}

5.1. Denoising by Empirical Mode Decomposition (EMD). EMD is designed to analyze nonlinear and nonstationary time series by decomposing them into intrinsic mode functions (IMFs) and residual [41]: 


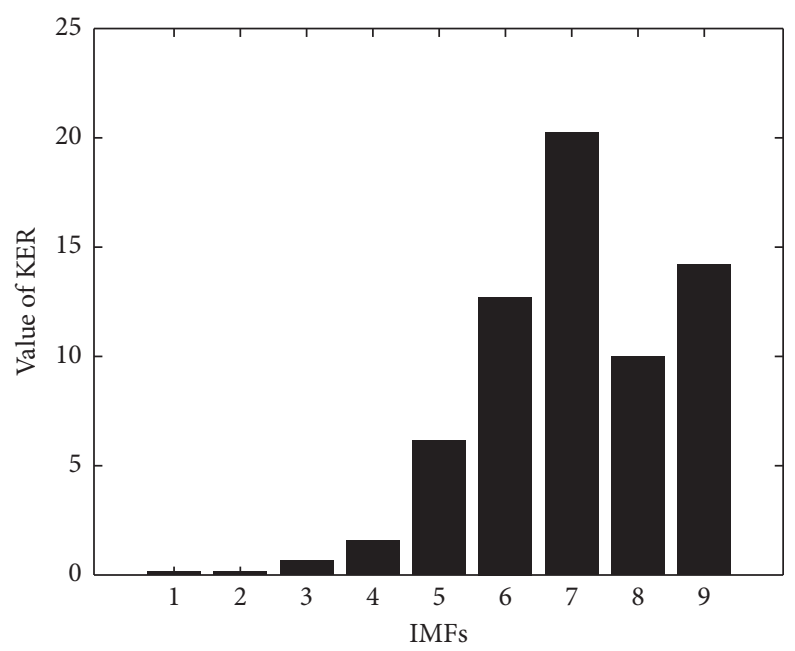

FIGURE 9: Values of KER for different IMFs.

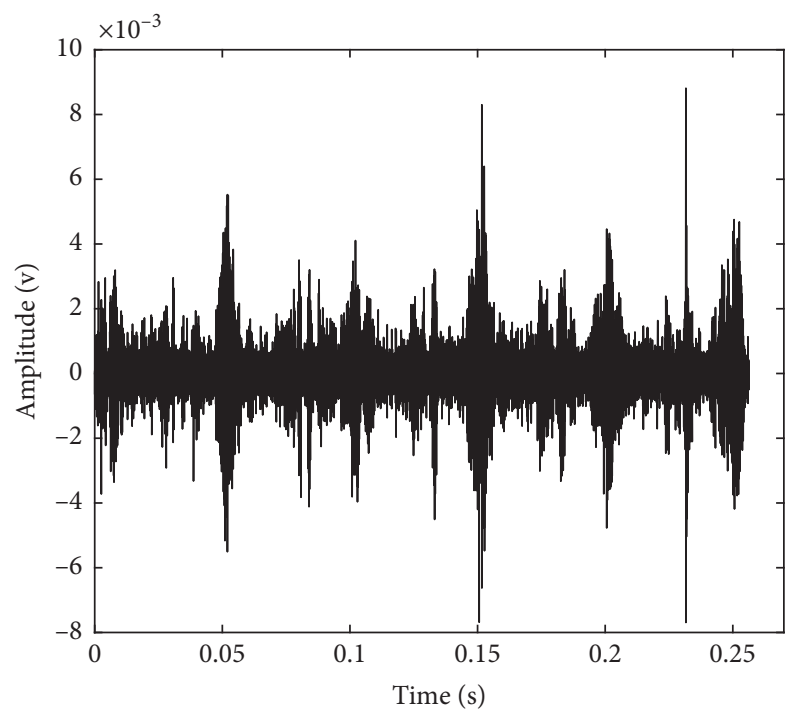

Figure 10: The denoised signal for small defect with condition of small load and speed of $600 \mathrm{rpm}$.

$$
\varphi(t)=\sum_{j=1}^{n} c_{j}(t)+r_{n}(t),
$$

where $c_{j}(t)$ and $r_{n}(t)$ are the $i$ th IMF and the trend term (residue) obtained by decomposition of the original signal $\varphi(t)$. For example, for a small defect with condition of small load and speed of $600 \mathrm{rpm}$, the EMD results are shown in Figure 8.

As you can see, the signal is decomposed into several IMFs, but in this level the proper IMF should be chosen; however, selecting the proper IMF, which shows the faulty state, is not easy and some criteria should be investigated to choose the proper IMF.

5.2. The Proposed Method. One of the most effective ways in diagnosis and fault detection of bearings is to monitor the value of the kurtosis of the acquired signal [42]. Entropy is also commonly used in signal processing and condition monitoring $[43,44]$. Adding a little noise to a signal will cause a significant change in the value of entropy. Thus, KER can be appropriate for identifying the signal components with the highest fault characteristics.

For selecting the denoised signal, in this paper, at first in the preprocessing stage, smoothing based on moving average method has been carried out on the raw signal for better results in signal decomposition especially for low SNR signals. After that, the obtained signals were decomposed by EMD, then KER values of all IMFs were calculated, and the highest value was selected as a desired signal. For the test with small defect with condition of small load and speed of $600 \mathrm{rpm}$, the KER values of all IMFs are shown in Figure 9.

As you can see, the IMF 7 has the highest KER, and it has little noise and is good for the fault detection. Accordingly, the denoised signal is depicted in Figure 10.

This process has also been done for other signals, and the results are shown in Figure 11. 


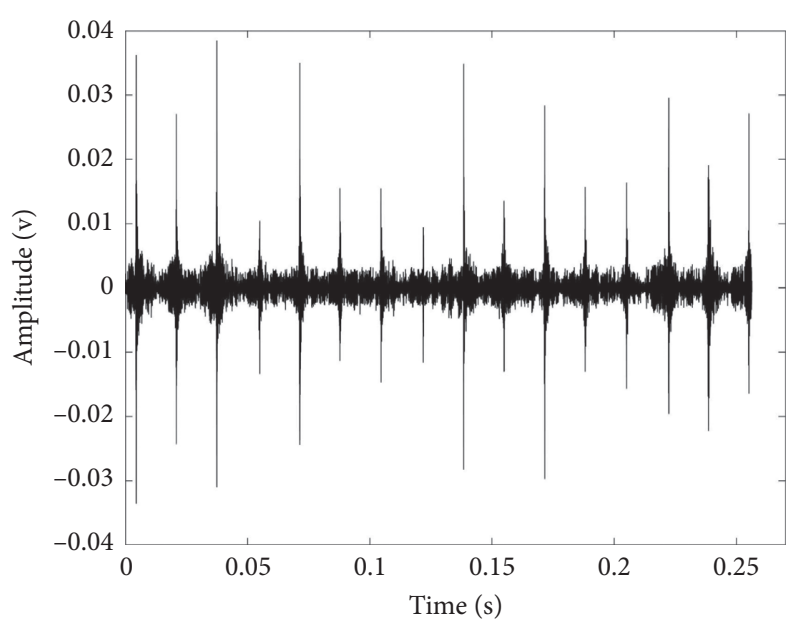

(a)

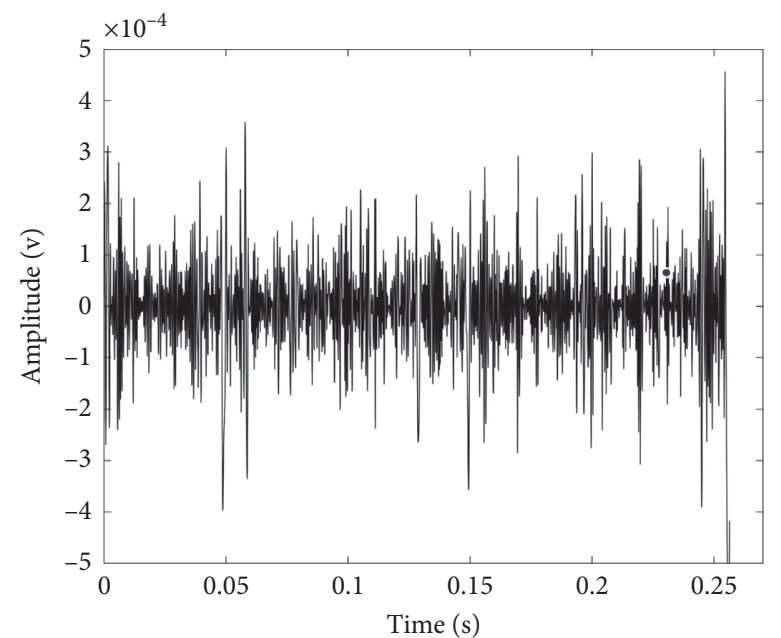

(c)

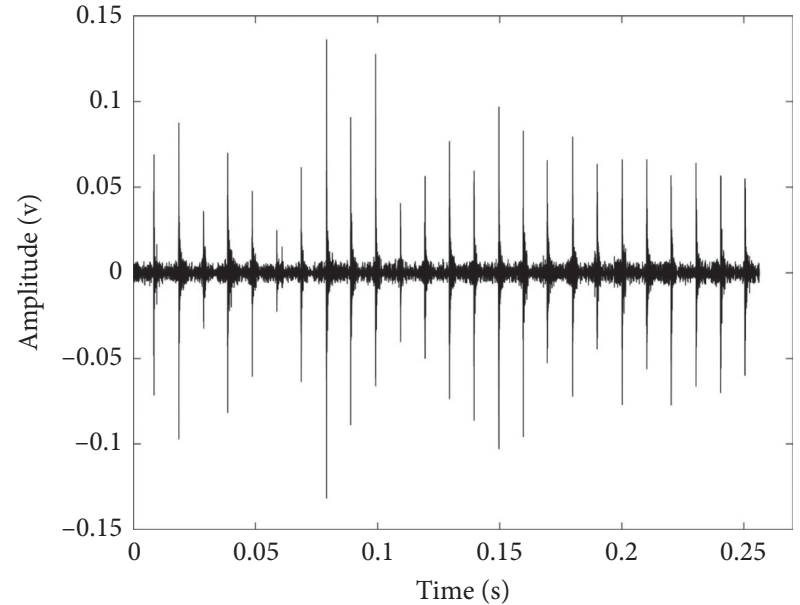

(b)

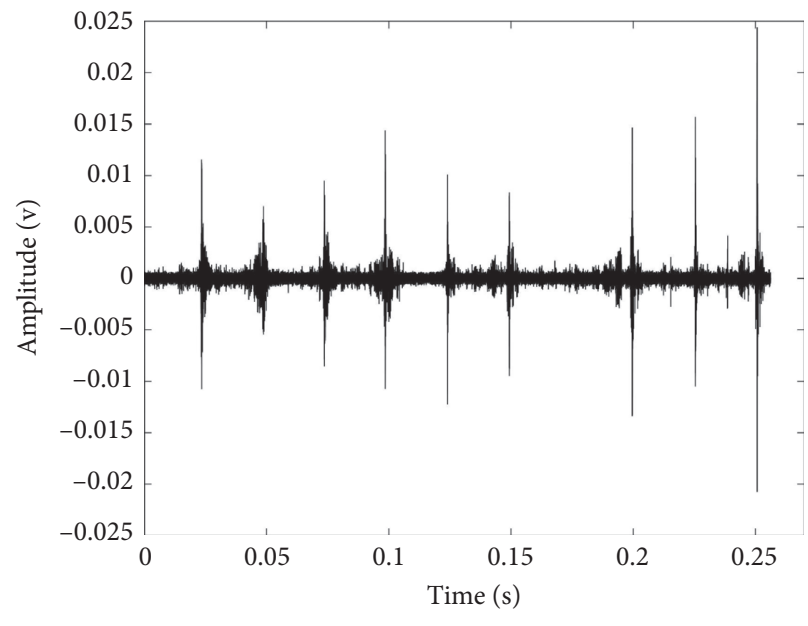

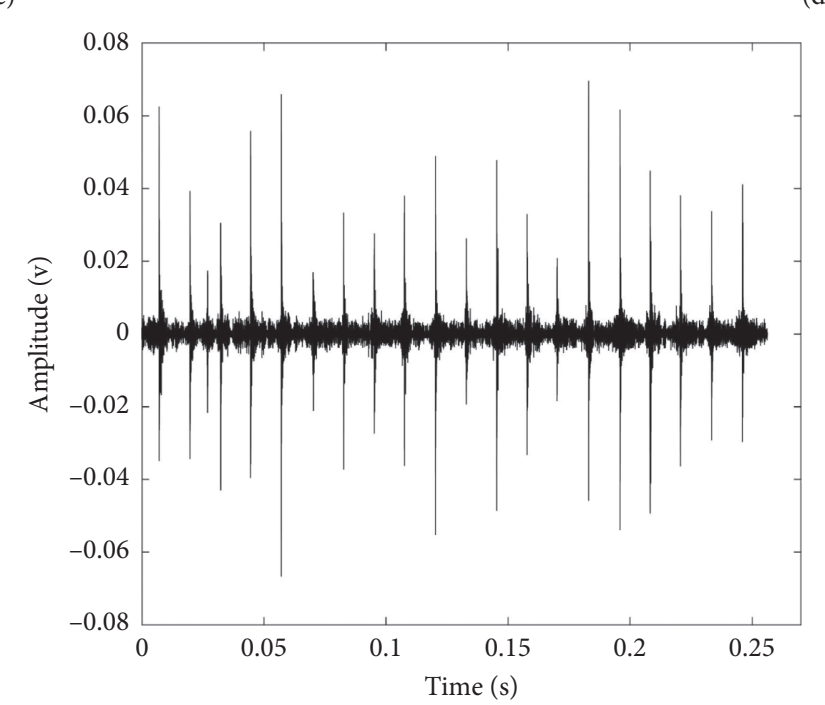

(e)

Figure 11: The denoised signals for other conditions. (a) Small defect, medium-low load, and $900 \mathrm{rpm}$. (b) Small defect, medium-high load, and $1500 \mathrm{rpm}$. (c) Large defect, small load, and $600 \mathrm{rpm}$. (d) Large defect, medium-high load, and $600 \mathrm{rpm}$. (e) Large defect, high load, and $1200 \mathrm{rpm}$. 
TABLE 4: Frequencies of the bearing in different speeds.

\begin{tabular}{lcccc}
\hline Shaft speed $(\mathrm{rpm})$ & 600 & 900 & 1200 & 1500 \\
\hline$F_{\mathrm{BPFO}}(\mathrm{Hz})$ & 40.272 & 60.408 & 80.543 & 100.679 \\
$F_{\mathrm{BPFI}}(\mathrm{Hz})$ & 59.728 & 89.592 & 119.457 & 149.321 \\
\hline
\end{tabular}

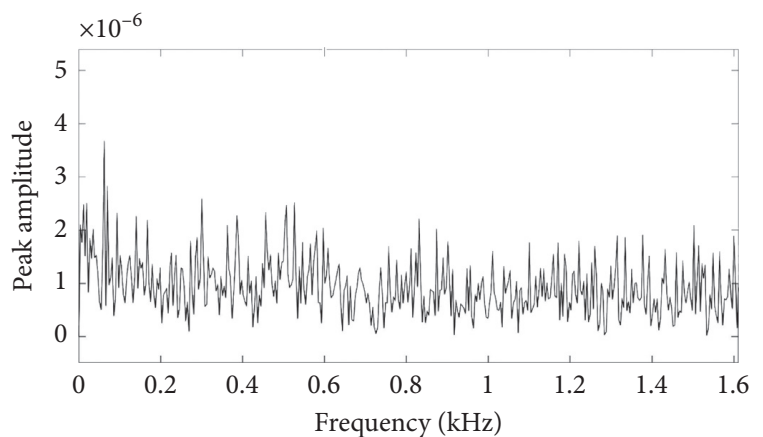

(a)

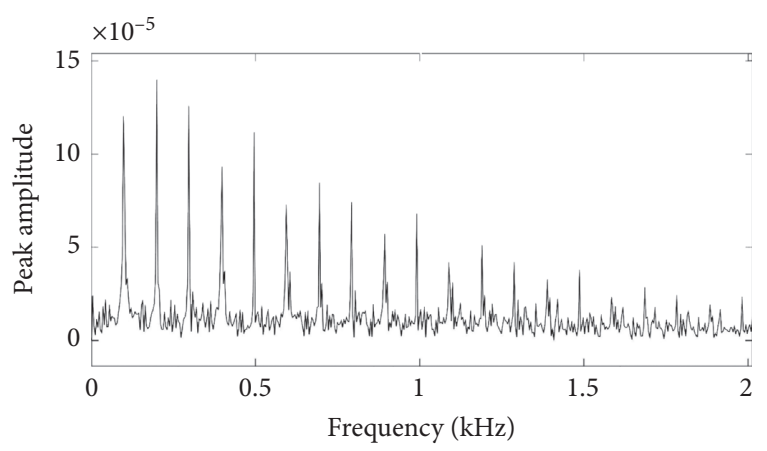

(c)

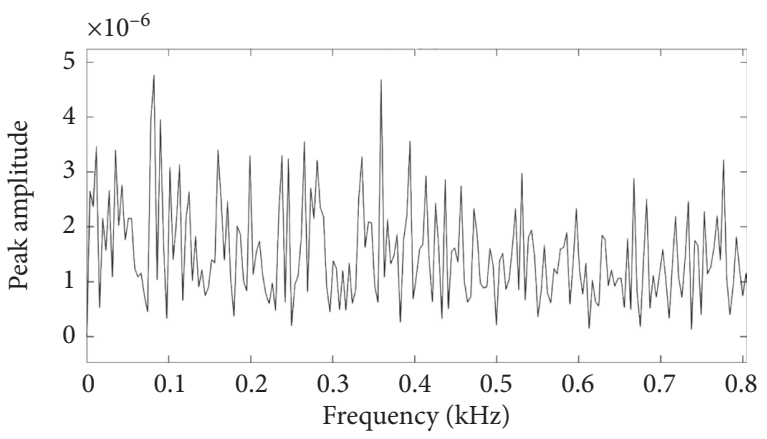

(e)

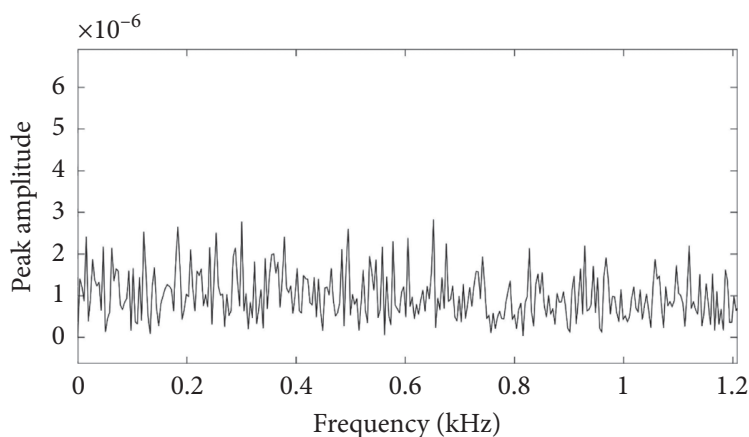

(b)

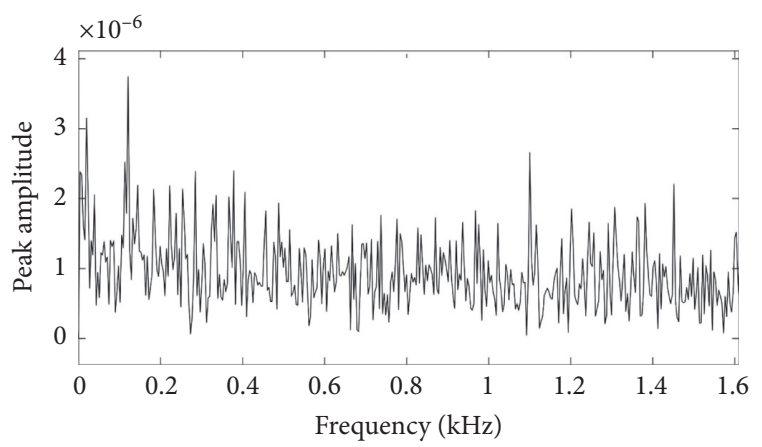

(d)

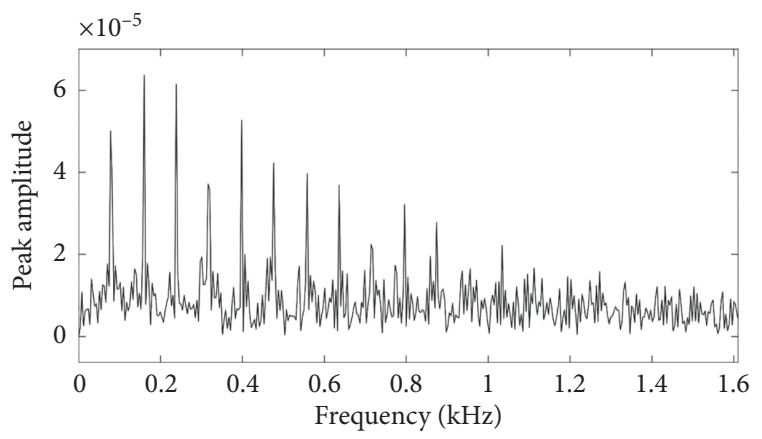

(f)

Figure 12: Envelope spectrum of signals before denoising. (a) Small defect, small load, and $600 \mathrm{rpm}$. (b) Small defect, medium-low load, and $900 \mathrm{rpm}$. (c) Small defect, medium-high load, and $1500 \mathrm{rpm}$. (d) Large defect, small load, and $600 \mathrm{rpm}$. (e) Large defect, medium-high load, and $600 \mathrm{rpm}$. (f) Large defect, high load, and $1200 \mathrm{rpm}$.

As you can see, by the proposed method, signals are improved, and noise is reduced.

\section{Fault Detection}

There are different methods for detecting the location of fault in the components of the REBs by extracting the characteristic defect frequency. The envelope spectra are a common tool for this purpose. The characteristic defect frequencies $\mathrm{F}_{\mathrm{BPFO}}$ and $\mathrm{F}_{\mathrm{BPFI}}$ are those of the outer race and inner race, respectively. They can be theoretically calculated as follows [45]:

$$
\begin{gathered}
F_{\mathrm{BPFO}}=\frac{n_{\mathrm{b}}}{2} f_{\mathrm{s}}\left(1-\frac{d_{\mathrm{b}} \cos \alpha}{d_{\mathrm{p}}}\right), \\
F_{\mathrm{BPFI}}=\frac{n_{\mathrm{b}}}{2} f_{\mathrm{s}}\left(1+\frac{d_{\mathrm{b}} \cos \alpha}{d_{\mathrm{p}}}\right),
\end{gathered}
$$




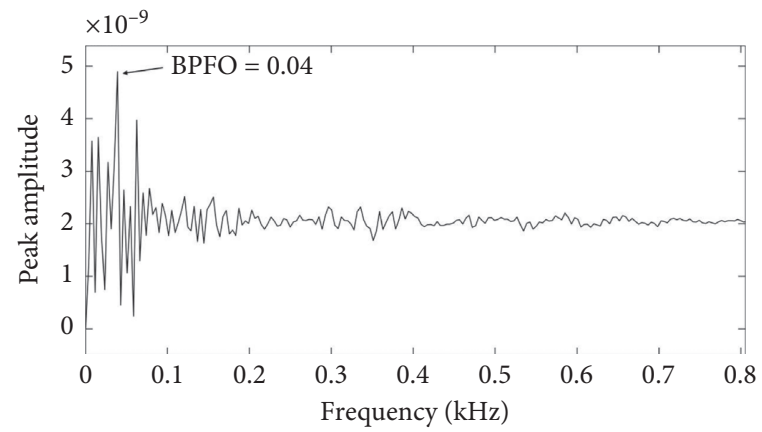

(a)

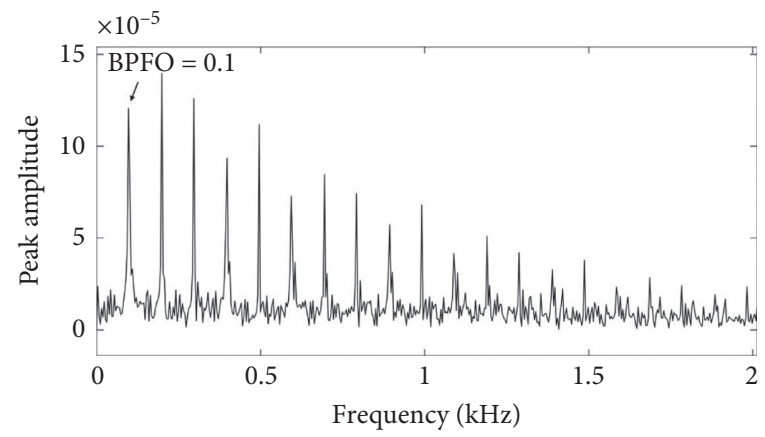

(c)

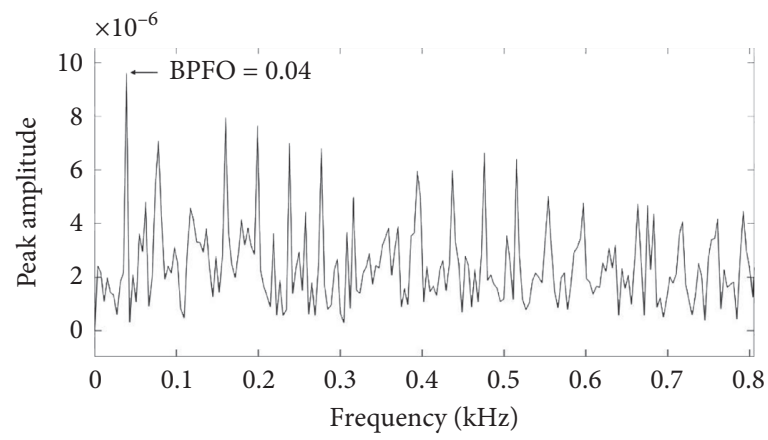

(e)

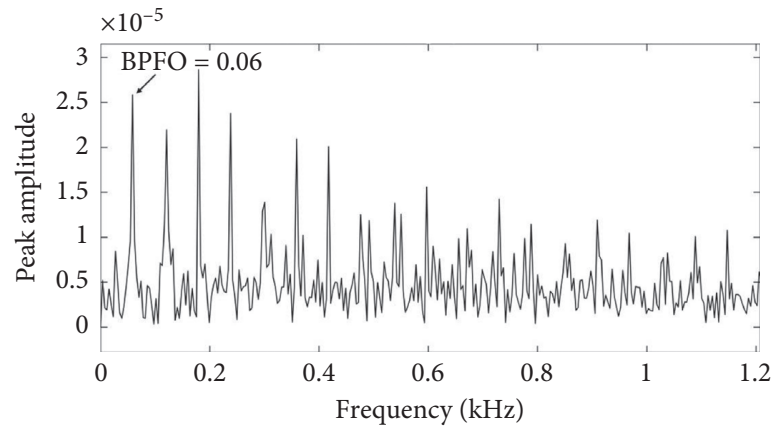

(b)

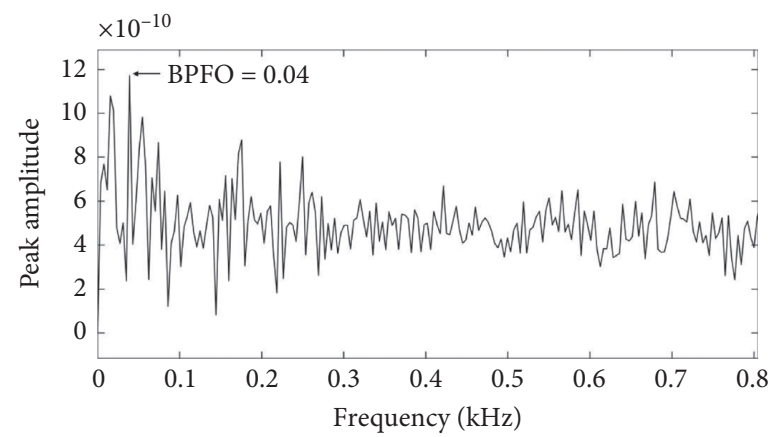

(d)

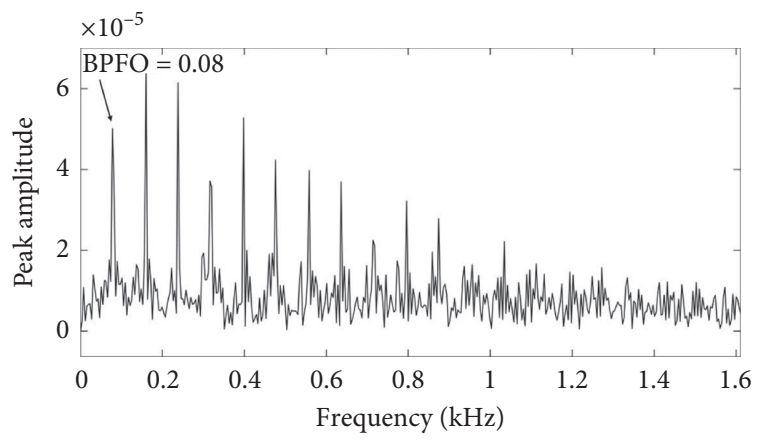

(f)

Figure 13: Envelope spectrum of signals after denoising. (a) Small defect, small load, and $600 \mathrm{rpm}$. (b) Small defect, medium-low load, and $900 \mathrm{rpm}$. (c) Small defect, medium-high load, and $1500 \mathrm{rpm}$. (d) Large defect, small load, and $600 \mathrm{rpm}$. (e) Large defect, medium-high load, and $600 \mathrm{rpm}$. (f) Large defect, high load, and $1200 \mathrm{rpm}$.

where $f_{\mathrm{s}}$ is the frequency of the shaft, $n_{\mathrm{b}}$ is the number of rolling elements, $d_{\mathrm{b}}$ is the ball diameter, $d_{\mathrm{p}}$ is the pitch diameter, and $\alpha$ is the contact angle. Table 4 shows the calculated characteristic frequencies of the bearing in different speeds.

In order to extract the characteristic defect frequency via $\mathrm{AE}$ signals, the envelope spectrum of the signals before applying the proposed method for denoising is depicted in Figure 12, and then the denoised signals by the proposed method are presented in Figure 13 for outer race at different operational conditions.

As can be seen from the above figures and compared with theoretically characteristic defect frequency (Table 4), this method detects faults successfully. Even in conditions with low SNR (Figures 7(a) and 7(d)) as shown in Figure 13, it can detect faults on both inner and outer race with good accuracy.

6.1. Faults on Inner Race. The process of detecting defects on inner race, in a similar way to outer race, is done. For example, for small defect with medium-low load and speed of $900 \mathrm{rpm}$, the process is depicted in Figure 14.

Envelope spectrum for other datasets, where faults are in inner race, at different operating conditions before and after applying the proposed method is depicted in Figures 15 and 16 , respectively.

As can be seen from above figures and compared with theoretically characteristic defect frequency (Table 4), this method detects faults successfully. 


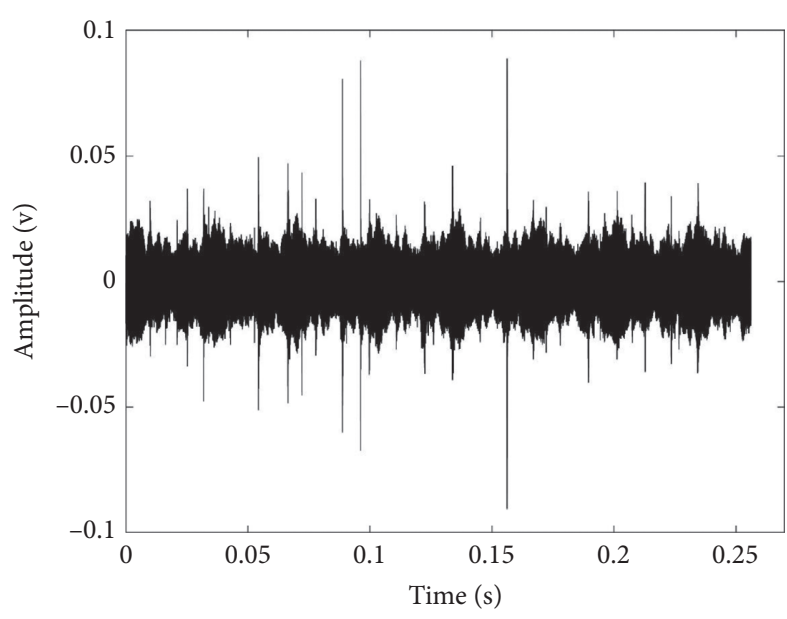

(a)

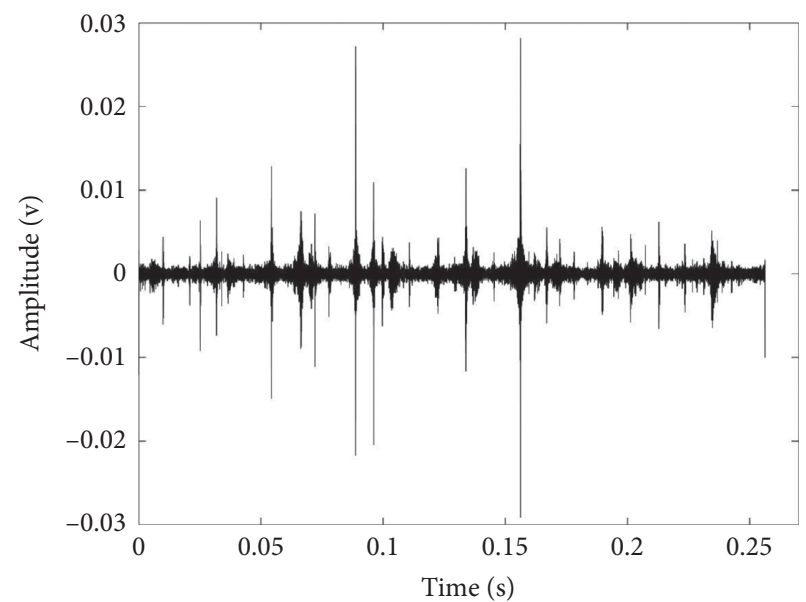

(b)

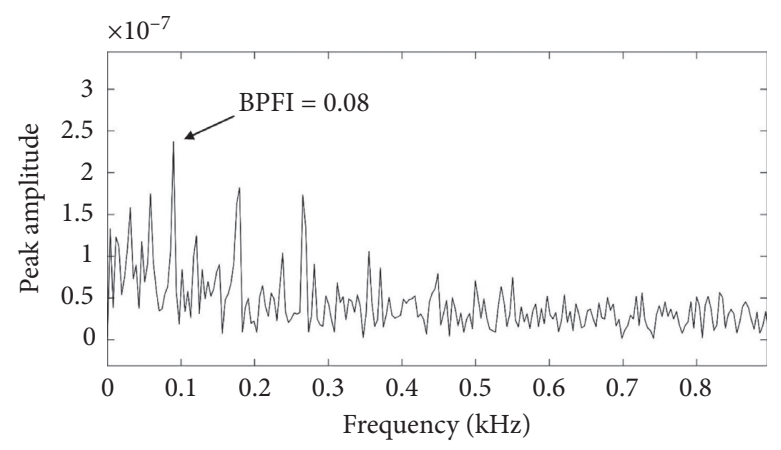

(c)

Figure 14: Fault detection on inner race. (a) Raw signal. (b) Denoised signal. (c) Envelope spectrum and fault detection.

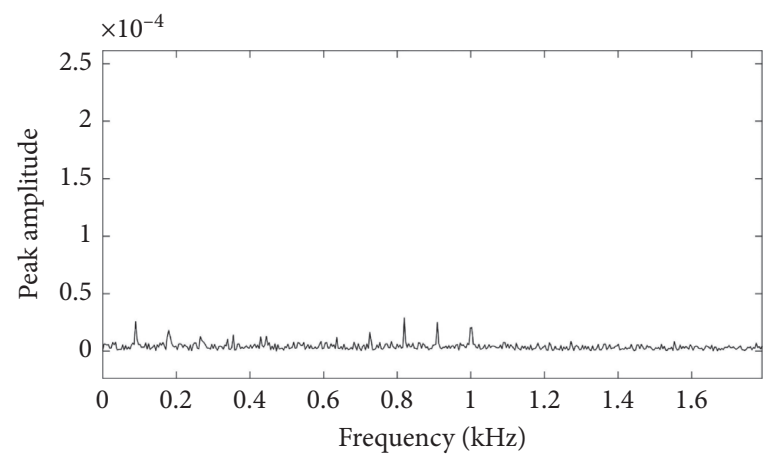

(a)

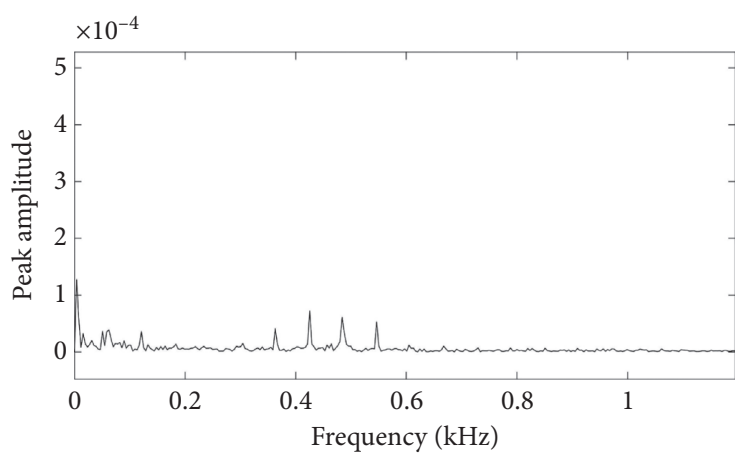

(c)

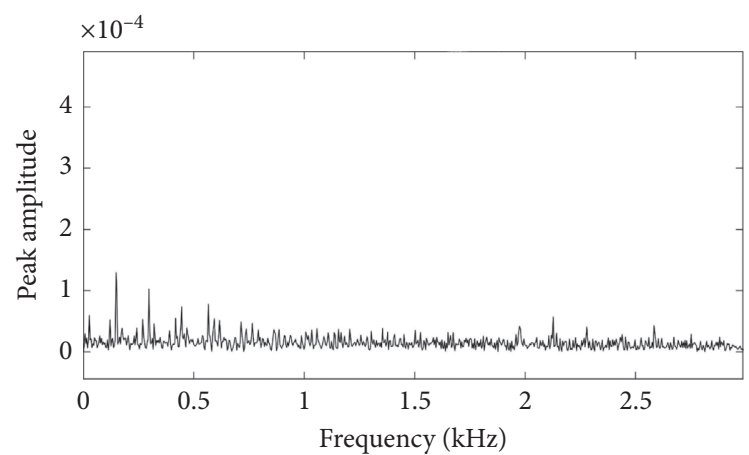

(b)

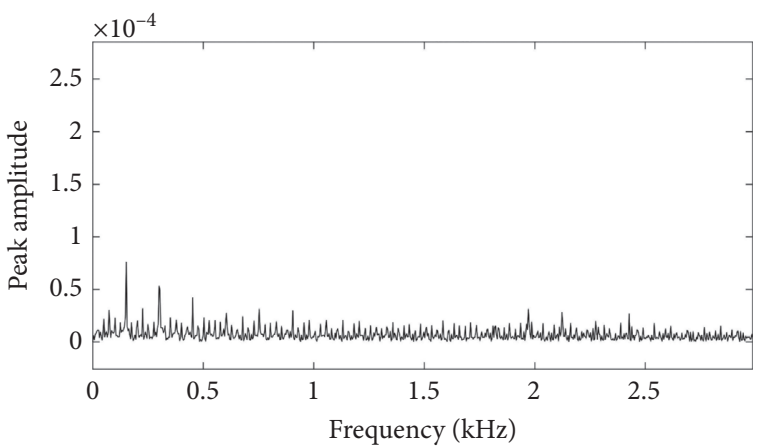

(d)

Figure 15: Continued. 


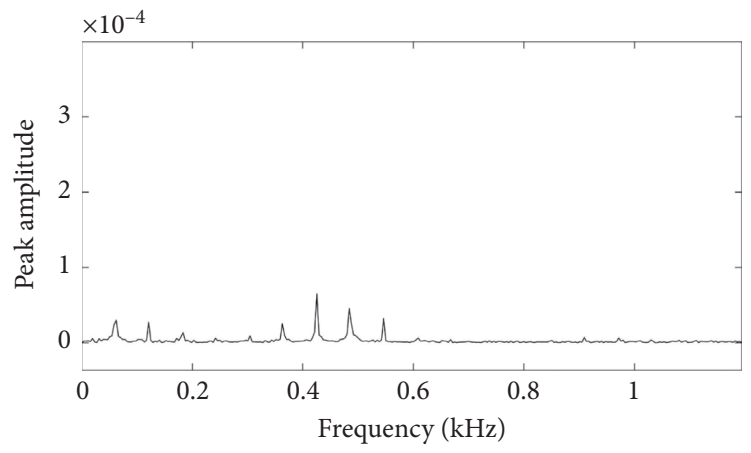

(e)

FiguRe 15: Envelope spectrum of signals before denoising. (a) Small defect, medium-high load, and $1500 \mathrm{rpm}$. (b) Small defect, high load, and $600 \mathrm{rpm}$. (c) Large defect, small load, and $1500 \mathrm{rpm}$. (d) Large defect, medium-low load, and $1200 \mathrm{rpm}$. (e) Large defect, high load, and $600 \mathrm{rpm}$.

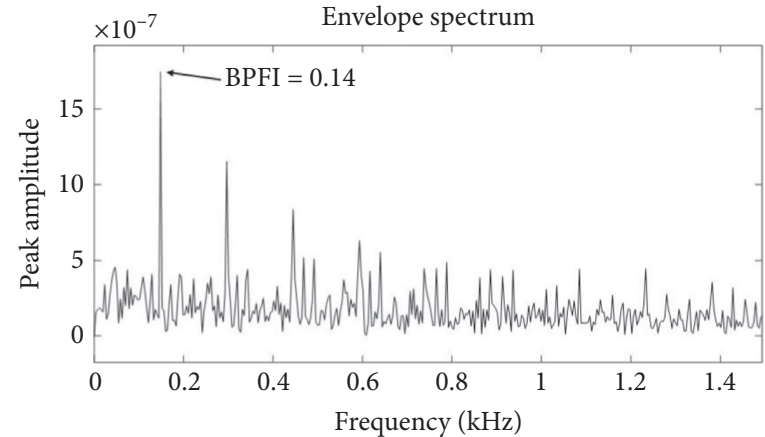

(a)

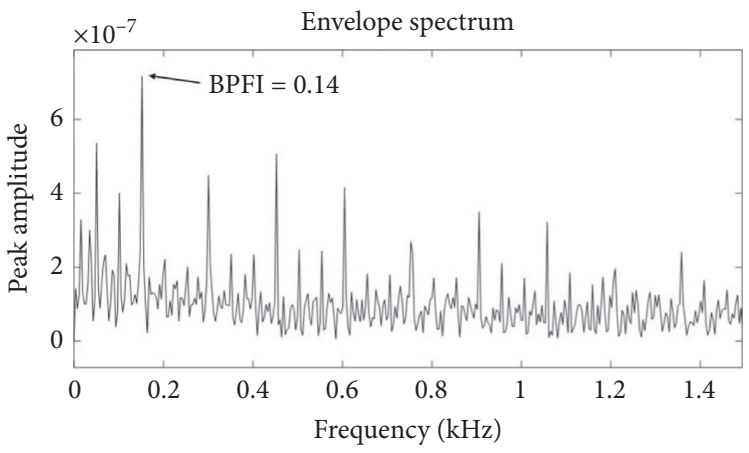

(c)

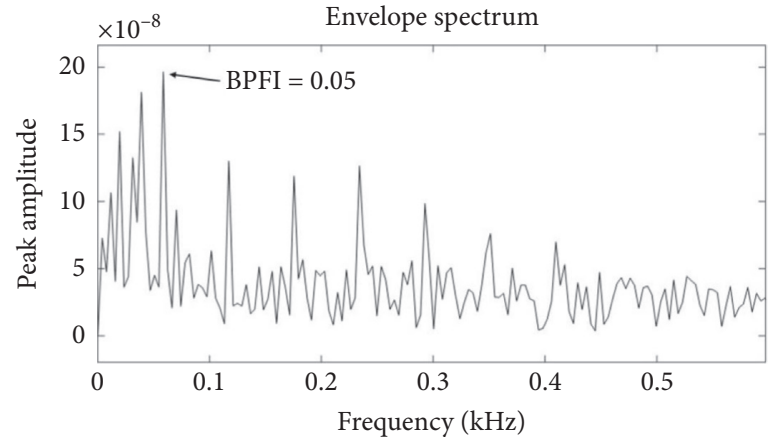

(b)

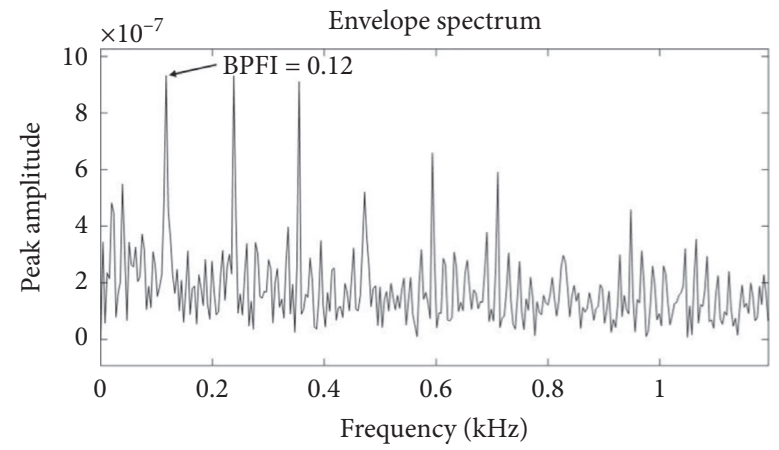

(d)

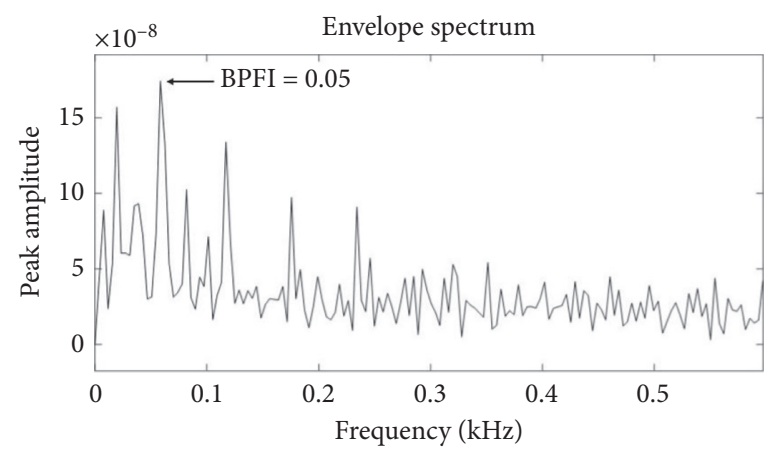

(e)

FIGURE 16: Envelope spectrum of signals after denoising. (a) Small defect, medium-high load, and $1500 \mathrm{rpm}$. (b) Small defect, high load, and $600 \mathrm{rpm}$. (c) Large defect, small load, and $1500 \mathrm{rpm}$. (d) Large defect, medium-low load, and $1200 \mathrm{rpm}$. (e) Large defect, high load, and $600 \mathrm{rpm}$. 


\section{Conclusion}

This paper is intended to present an efficient and accurate method, AE method, for fault detection of REBs. For this purpose, an angular contact bearing was selected and an experimental study was carried out.

In this work, AE signals were gathered and then denoised by EMD, and appropriate IMF based on KER wass selected. KER indicator was able to successfully choose optimized IMF with the highest SNR. Then, defect frequencies were extracted by envelope spectrum of desired signal, and defects were detected. The experiment was performed under different conditions such as defect size, loading, and rotational speed.

The results demonstrate the effectiveness and robustness of the proposed method in detecting defects on both inner and outer race even in the cases of small defects and low SNR AE signals. The proposed method has been implemented successfully for a defect size of $0.3 \mathrm{~mm}$ (small defect) on the outer race with a small load and speed of $600 \mathrm{rpm}$, and it showed a fault frequency of $0.04 \mathrm{kHz}$. Furthermore, the proposed method precisely detected the small defect on the inner race with medium-low load and speed of $900 \mathrm{rpm}$.

Detection of small defects with a small load and small speed could be one of the advantages of the proposed method. However, the method still needs to be implemented on different conditions and cases and for higher loads with higher speeds. Moreover, there are some efforts to improve the performance of diagnosing; for example, if the speed of the machine is not constant, the current approach shall be modified. In the future, the proposed method can be investigated in terms of the diagnosis of other rotating components such as gears.

\section{Data Availability}

The data used to support the findings of this study are available from the corresponding author upon request.

\section{Conflicts of Interest}

The authors declare that there are no conflicts of interest regarding the publication of this paper.

\section{Acknowledgments}

Experimental tests of this research were conducted in the IPCO Company, so the authors express their thanks to the company for their cooperation.

\section{References}

[1] T. A. Harris, Rolling Bearing Analysis, John Wiley and Sons, Hoboken, NJ, USA, 2001.

[2] http://www.angularcontact-ballbearing.com.

[3] I. Howard, A Review of Rolling Element Bearing Vibration Detection, Diagnosis and Prognosis, Defence Science and Technology Organization, Canberra, Australia, 1994.

[4] S. Singh, C. Q. Howard, and C. H. Hansen, "An extensive review of vibration modelling of rolling element bearings with localised and extended defects," Journal of Sound and Vibration, vol. 357, pp. 300-330, 2015.

[5] S. Nandi, H. A. Toliyat, and X. Li, "Condition monitoring and fault diagnosis of electrical motors-A review," IEEE Transactions on Energy Conversion, vol. 20, no. 4, pp. 719-729, 2005.

[6] A. M. Al-Ghamd and D. Mba, "A comparative experimental study on the use of acoustic emission and vibration analysis for bearing defect identification and estimation of defect size," Mechanical Systems and Signal Processing, vol. 20, no. 7, pp. 1537-1571, 2006.

[7] T. Sako and O. Yoshie, "Diagnostic method of low speed rolling element bearing using AE envelope waveform," in Proceedings of the TENCON 2010-2010 IEEE Region 10 Conference, pp. 724-729, IEEE, Fukuoka, Japan, November 2010.

[8] H. L. Balderston, "The detection of incipient failure in bearings," Mater Evaluation, vol. 27, no. 6, pp. 121-128, 1969.

[9] T. Yoshioka and T. Fujiwara, "A new acoustic emission source locating system for the study of rolling contact fatigue," Wear, vol. 81, no. 1, pp. 183-186, 1982.

[10] L. M. Rogers, "The application of vibration signature analysis and acoustic emission source location to on-line condition monitoring of anti-friction bearings," Tribology International, vol. 12, no. 2, pp. 51-58, 1979.

[11] N. Tandon and B. C. Nakra, "The application of the soundintensity technique to defect detection in rolling-element bearings," Applied Acoustics, vol. 29, no. 3, pp. 207-217, 1990.

[12] N. Tandon and A. Choudhury, "A review of vibration and acoustic measurement methods for the detection of defects in rolling element bearings," Tribology International, vol. 32, no. 8, pp. 469-480, 1999.

[13] A. Choudhury and N. Tandon, "Application of acoustic emission technique for the detection of defects in rolling element bearings," Tribology International, vol. 33, no. 1, pp. 39-45, 2000.

[14] D. Mba, "Acoustic emissions and monitoring bearing health," Tribology Transactions, vol. 46, no. 3, pp. 447-451, 2003.

[15] D. Mba and R. B. Rao, "Development of acoustic emission technology for condition monitoring and diagnosis of rotating machines; bearings, pumps, gearboxes, engines and rotating structures," 2006.

[16] Z. Rahman, H. Ohba, T. Yoshioka, and T. Yamamoto, "Incipient damage detection and its propagation monitoring of rolling contact fatigue by acoustic emission," Tribology International, vol. 42, no. 6, pp. 807-815, 2009.

[17] J. Z. Sikorska and D. Mba, "Challenges and obstacles in the application of acoustic emission to process machinery," Proceedings of the Institution of Mechanical Engineers, Part E: Journal of Process Mechanical Engineering, vol. 222, no. 1, pp. 1-19, 2008.

[18] V. Kappatos and E. Dermatas, "Crack detection in noisy environment including raining conditions," Aircraft Engineering and Aerospace Technology, vol. 79, no. 2, pp. 163-169, 2007.

[19] B. Eftekharnejad, M. R. Carrasco, B. Charnley, and D. Mba, "The application of spectral kurtosis on acoustic emission and vibrations from a defective bearing," Mechanical Systems and Signal Processing, vol. 25, no. 1, pp. 266-284, 2011.

[20] R. Li and D. He, "Rotational machine health monitoring and fault detection using EMD-based acoustic emission feature quantification," IEEE Transactions on Instrumentation and Measurement, vol. 61, no. 4, pp. 990-1001, 2012.

[21] C. Lu, Q. Sun, L. Tao, H. Liu, and C. Lu, "Bearing health assessment based on chaotic characteristics," Shock and Vibration, vol. 20, no. 3, pp. 519-530, 2013. 
[22] J. Antoni and R. B. Randall, "The spectral kurtosis: application to the vibratory surveillance and diagnostics of rotating machines," Mechanical Systems and Signal Processing, vol. 20, no. 2, pp. 308-331, 2006.

[23] C. T. Yiakopoulos and I. A. Antoniadis, "Wavelet based demodulation of vibration signals generated by defects in rolling element bearings," Shock and Vibration, vol. 9, no. 6, pp. 293-306, 2002.

[24] G. G. Amiri and A. Asadi, "Comparison of different methods of wavelet and wavelet packet transform in processing ground motion records," International Journal of Civil Engineering, vol. 7, no. 4, pp. 248-257, 2009.

[25] R. J. Hao, Z. P. Feng, and F. L. Chu, "Defects diagnosis of bearing by means of acoustic emission and continuous wavelet transform," Key Engineering Materials, vol. 413-414, pp. 651-657, 2009.

[26] H. A. Parizi, M. Kafil, M. Ghayour, and S. Z. Rad, "Fault diagnosis of slow-speed rolling ele-ment bearings with the use of acoustic emission and wavelet packet," in Proceedings of the 21st International Congress on Sound and Vibration, pp. 1-8, Beijing, China, July 2014.

[27] A. P. Rodrigues and G. DaAZMello, "Selection of mother wavelet for wavelet analysis of vibration signals in machining," Journal of Mechanical Engineering and Automation, vol. 6, no. 5A, pp. 81-85, 2016.

[28] F. Hemmati, W. Orfali, and M. S. Gadala, "Roller bearing acoustic signature extraction by wavelet packet transform, applications in fault detection and size estimation," Applied Acoustics, vol. 104, pp. 101-118, 2016.

[29] J. L. F. Chacon, V. Kappatos, W. Balachandran, and T.-H. Gan, "A novel approach for incipient defect detection in rolling bearings using acoustic emission technique," Applied Acoustics, vol. 89, pp. 88-100, 2015.

[30] L. Cheng, S. Fu, H. Zheng, Y. Huang, and Y. Xu, "Fault identification and severity assessment of rolling element bearings based on EMD and fast kurtogram," Journal of Vibroengineering, vol. 18, no. 6, 2016.

[31] D. Liu, J. Tao, A. Luo, and Q. Wang, “An optimized kurtogram method for early fault detection of rolling element bearings using acoustic emission," in Proceedings of the 2018 International Conference on Information Systems and Computer Aided Education (ICISCAE), pp. 365-370, IEEE, Changchun, China, July 2018.

[32] V. Bajaj and R. B. Pachori, "Classification of seizure and nonseizure EEG signals using empirical mode decomposition," IEEE Transactions on Information Technology in Biomedicine, vol. 16, no. 6, pp. 1135-1142, 2012.

[33] A. Priya, P. Yadav, S. Jain, and V. Bajaj, "Efficient method for classification of alcoholic and normal EEG signals using EMD," The Journal of Engineering, vol. 2018, no. 3, pp. 166-172, 2018.

[34] S. Jain, V. Bajaj, and A. Kumar, "Riemann Liouvelle fractional integral based empirical mode decomposition for ECG denoising," IEEE Journal of Biomedical and Health Informatics, vol. 22, no. 4, pp. 1133-1139, 2018.

[35] Y. He, X. Zhang, and M. I. Friswell, "Defect diagnosis for rolling element bearings using acoustic emission," Journal of Vibration and Acoustics, vol. 131, no. 6, Article ID 061012, 2009.

[36] N. Tandon, K. M. Ramakrishna, and G. S. Yadava, "Condition monitoring of electric motor ball bearings for the detection of grease contaminants," Tribology International, vol. 40, no. 1, pp. 29-36, 2007.

[37] S. A. Niknam, V. Songmene, and Y. J. Au, "The use of acoustic emission information to distinguish between dry and lubricated rolling element bearings in low-speed rotating machines," The International Journal of Advanced Manufacturing Technology, vol. 69, no. 9-12, pp. 2679-2689, 2013.

[38] K. Wang, X. Liu, X. Wu, and Z. Zhu, "Monitoring the lack of grease condition of rolling bearing using acoustic emission," International Journal of Modelling, Identification and Control, vol. 31, no. 1, pp. 94-102, 2019.

[39] D. Dyer and R. M. Stewart, "Detection of rolling element bearing damage by statistical vibration analysis," Journal of Mechanical Design, vol. 100, no. 2, pp. 229-235, 1978.

[40] H. Qiu, J. Lee, J. Lin, and G. Yu, "Wavelet filter-based weak signature detection method and its application on rolling element bearing prognostics," Journal of Sound and Vibration, vol. 289, no. 4-5, pp. 1066-1090, 2006.

[41] N. E. Huang, Z. Shen, S. R. Long et al., "The empirical mode decomposition and the Hilbert spectrum for nonlinear and non-stationary time series analysis," Proceedings of the Royal Society of London. Series A: Mathematical, Physical and Engineering Sciences, vol. 454, no. 1971, pp. 903-995, 1998.

[42] A. R. Bastami, A. Aasi, and H. A. Arghand, "Estimation of remaining useful life of rolling element bearings using wavelet packet decomposition and artificial neural network," Iranian Journal of Science and Technology, Transactions of Electrical Engineering, vol. 43, no. 1, pp. 233-245, 2019.

[43] K. Deák, T. Mankovits, and I. Kocsis, "Optimal wavelet selection for the size estimation of manufacturing defects of tapered roller bearings with vibration measurement using Shannon entropy criteria," Strojniski Vestnik/Journal of Mechanical Engineering, vol. 63, no. 1, 2017.

[44] J.-D. Wu and C.-H. Liu, "An expert system for fault diagnosis in internal combustion engines using wavelet packet transform and neural network," Expert Systems with Applications, vol. 36, no. 3, pp. 4278-4286, 2009.

[45] B. Kilundu, X. Chiementin, J. Duez, and D. Mba, "Cyclostationarity of Acoustic Emissions (AE) for monitoring bearing defects," Mechanical Systems and Signal Processing, vol. 25, no. 6, pp. 2061-2072, 2011. 